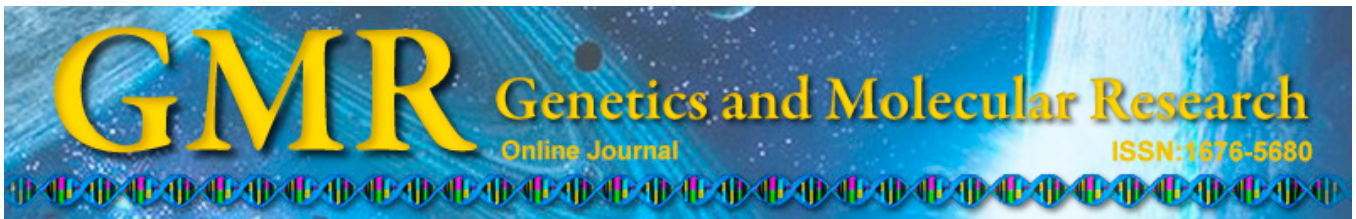

\title{
Immunomodulatory and clinical effects of the "tiaomian III decoction" in patients with blood blocking antibody deficiency and recurrent spontaneous abortion
}

\author{
H.Y. Gao*, E.X. Tao*, Y. Wang, Q.A. Yue, C.E. Ren and L.F. Yan \\ Tradicional Chinese Medicine Department, \\ Affiliated Hospital of Weifang Medical University, Weifang, Shandong, China \\ *These authors contributed equally to this study. \\ Corresponding author: H.Y. Gao \\ E-mail: gaohanyifml@126.com \\ Genet. Mol. Res. 14 (2): 3421-3425 (2015) \\ Received May 5, 2014 \\ Accepted October 28, 2014 \\ Published April 15, 2015 \\ DOI http://dx.doi.org/10.4238/2015.April.15.5
}

\begin{abstract}
We studied the immunomodulatory and clinical effects of the empirical formula "tiaomian III decoction" on maternal blood blocking antibody deficiency and recurrent spontaneous abortion. Sixty-one patients with blocking antibody deficiency were divided in the experimental group $(\mathrm{N}=31)$, who took tiaomian III decoction, and the control group $(\mathrm{N}=30)$, who received active immunotherapy with paternal lymphocytes; both treatments lasted 3 months. Blocking antibodies, anti-idiotypic antibodies, interleukin, T-lymphocyte subsets, and macrophage colony-stimulating factor (M-CSF) were tested. After treatment, the positive conversion rate reached 87.1 and $86.7 \%$ in the experimental and control groups, respectively. After treatment, CD4 levels decreased while CD8 levels increased in both groups. The $\mathrm{CD} 4 / \mathrm{CD} 8$ ratio was higher than normal and increased significantly from pre-treatment $(\mathrm{P}<0.05)$. IL-10 and M-CSF levels increased
\end{abstract}


significantly in both groups $(\mathrm{P}<0.05)$. The 1 -year conception rates of the experimental and control groups were 58.1 and $46.7 \%$, respectively $(\mathrm{P}<0.05)$. The results show the tiaomian III decoction can increase the positive conversion rate of maternal blocking antibodies and promote the production of IL-10 and M-CSF. Thus, it strengthens the maternal body's protection of the fetus and maintenance of conception. The higher conception rate of the experimental group demonstrates the positive clinic efficacy of the tiaomian III decoction on maternal blood blocking antibody deficiency and recurrent spontaneous abortion.

Key words: Recurrent spontaneous abortion; Blocking antibody deficiency; Anti-idiotype deficiency; Interleukin; T-lymphocyte subsets

\section{INTRODUCTION}

Recurrent spontaneous abortion (RSA) is common gynecopathy frequently termed knotty disease. Besides hereditary, endocrine, and reproductive anatomical factors as well as infection, about $40-65 \%$ of cases are associated with immunization; $54.9 \%$ involve maternal blood blocking antibody deficiency (Yu et al. 2002). Since 2007, we have treated 31 patients with maternal blood antibody deficiency RSA by using an empirical formula from traditional Chinese medicine called "tiaomian III decoction," which produces a positive clinical effect.

\section{MATERIAL AND METHODS}

\section{Diagnostic criteria}

The main diagnostic criterion was patients with RSA lacking blocking antibody (Ab1) or anti-idiotypic antibodies (Ab2) $<5 \%$.

The inclusion criteria were as follows: 1) patients with RSA or no less than 2 stillbirths; 2) Ab1 and/or Ab2 <5\%; 3) semen from spouse tested normal; 4) informed consent from the patient and her spouse. The exclusion criteria were as follows: 1) RSA due to hereditary factors or ABO blood type incompatibility; 2) maternal family history of RSA caused by abnormalities of the endocrine system, sperm antibodies, zona pellucida antibodies, endometrial antibodies, reproductive organ anatomy, phospholipid antibodies, or lupus anticoagulant; 3) RSA due to infection caused by bacteria, virus, protozoa, mycoplasma, or chlamydia; 4) RSA caused by IgM-positive blood toxoplasma, rubella virus, cytomegalovirus, or herpes simplex virus.

\section{Therapy}

Patients in the experimental group took the tiaomian III decoction, which includes human placenta (taken with water), Epimedium, Morinda officinalis, Astragalus, Codonopsis pilosula, Cuscuta chinensis, Dipsacaceae, Eucommia cortex (Eucommiae), white peony root, roasted rhizoma Atractylodis Macrocephalae, Glycyrrhiza uralensis, and other herbs made into an oral solution with $1 \mathrm{~g}$ medicine per milliliter (Gao et al., 2012). Patients took $100 \mathrm{~mL}$ twice daily with $5 \mathrm{~g}$ human placenta 5 days per week. Meanwhile, patients in the control group 
took active immunotherapy involving paternal lymphocytes treatment. First, $50 \mathrm{~mL}$ peripheral fresh blood was taken from their spouse. The lymphocytes were isolated and placed into incubator together with $10 \mathrm{~mL}$ induced liquid for 3 days. Then, intradermal injections were made at multiple points on the forearm once every 3 weeks for 4 times. The treatment effects were analyzed after 3 months. When $\mathrm{Ab} 1$ and $\mathrm{Ab} 2$ are $>5 \%$, the patients can become pregnant. Furthermore, we traced the pregnancy rate within 1 year of treatment.

Blocking antibodies, anti-idiotypic antibodies, T-lymphocyte subsets, interleukin (IL)-10, and macrophage colony-stimulating factor (M-CSF) were analyzed.

\section{Statistical analysis}

The SPSS version 11.0 was used to analyze the data. The $\chi^{2}$ test and $t$-test were used to analyze categorical and numerical data, respectively.

\section{RESULTS}

After applying the inclusion and exclusion criteria, 61 patients with $\mathrm{Ab} 1 \mathrm{and} / \mathrm{or} \mathrm{Ab} 2$ $<5 \%$ were enrolled and randomly divided into 2 groups. The experimental group included 31 patients aged 26-35 years, with a mean disease duration of $4.5 \pm 1.6$ years (range 3-7 years). Meanwhile, the control group included 30 patients aged 25-36 years, with a mean disease duration of 4.6 \pm 1.6 years (range: $3-8$ years). There were no significant differences in the baseline characteristics of these groups $(\mathrm{P}>0.05)$. Furthermore, the normal control group included 10 healthy parous women aged 24-36 years with normal blocking antibodies (Ab1 and Ab2 $>5 \%$ ), who were tested for T- lymphocyte subsets (Ab2), IL-10, and M-CSF.

The positive conversion rates of the blocking antibodies of the 2 groups post-treatment are shown in Table 1. Post-treatment, the positive conversion rates of the experimental and control groups were 87.1 and $86.7 \%$, respectively.

The T-lymphocyte subsets in the peripheral blood of the 2 groups pre- and post-treatment are shown in Table 2.

CD4 levels were higher in the experimental and control groups than the normal group; meanwhile, CD8 levels were lower than normal group $(\mathrm{P}<0.05)$. This indicates that patients with RSA lack blocking antibody with disordered immune function characterized by elevated CD4 and depressed CD8.

Post-treatment, CD4 levels decreased in the experimental and control groups while CD8 levels increased. The CD4/CD8 ratio was higher than normal and increased significantly from pre-treatment $(\mathrm{P}<0.05)$. This indicates both treatments had a positive immune function regulating effect.

IL-10 and M-CSF levels pre- and post-treatment are shown in Table 3. There were no obvious differences in IL-10 or M-CSF levels between the experimental and control groups pre-treatment $(\mathrm{P}>0.05)$; both were significantly lower than those in the normal group $(\mathrm{P}<$ $0.05)$. This indicates patients with RSA lacking blocking antibodies have lower levels of IL-10 and M-CSF. Post-treatment, IL-10 and M-CSF levels increased significantly in both groups (P $<0.01$ ), indicating the secretion of IL-10 and M-CSF increased, which is helpful for maintaining pregnancy, in both groups.

Regarding pregnancy rate within 1 year, 18 patients in the experimental group became pregnant, and 2 became pregnant for longer than before, representing a pregnancy rate of $58.1 \%$. 
Meanwhile, in the control group, 16 patients became pregnant, 2 experienced spontaneous abortion, and 14 were pregnant for longer than before, representing a pregnancy rate of $46.7 \%$. Thus, patients in the experimental group were more likely to become pregnant within 1 year posttreatment. Furthermore, the decoction was safer, less toxic, and had fewer side effects.

Table 1. Comparison of blocking antibodies positive conversing rate of the two groups after treatment.

\begin{tabular}{lccc}
\hline Group & No. of patients & Negative (\%) & Positive (\%) \\
\hline Experimental group & 31 & $4(12.9)$ & $27(87.1)$ \\
Control group & 30 & $4(13.3)$ & $26(86.7)$ \\
\hline Ab1 and
\end{tabular}

$\mathrm{Ab} 1$ and/or $\mathrm{Ab} 2$ all $>5 \%$ is considered as positive; $\mathrm{Ab} 1 \mathrm{and} /$ or $\mathrm{Ab} 2<5 \%$ is negative.

Table 2. Comparison of T-lymphocyte cell subsets in peripheral blood of the two groups before and after treatment (means $\pm \mathrm{SD}$ ).

\begin{tabular}{|c|c|c|c|c|c|c|c|}
\hline \multirow[b]{2}{*}{ Group } & \multirow[b]{2}{*}{ No. of patients } & \multicolumn{3}{|c|}{ Before treatment } & \multicolumn{3}{|c|}{ After treatment } \\
\hline & & CD4 (\%) & CD8 (\%) & $\mathrm{CD} 4 / \mathrm{CD} 8$ & CD4 (\%) & CD8 (\%) & $\mathrm{CD} 4 / \mathrm{CD} 8$ \\
\hline Experimental group & 31 & $46.8 \pm 2.5$ & $22.2 \pm 1.6$ & $2.1 \pm 0.3$ & $40.3 \pm 2.2 *$ & $26.1 \pm 2.1 *$ & $1.6 \pm 0.5^{*}$ \\
\hline Control group & 30 & $46.5 \pm 2.6^{*}$ & $22.1 \pm 2.1^{*}$ & $2.0 \pm 0.2$ & $40.1 \pm 2.0$ & $26.2 \pm 1.2$ & $1.6 \pm 0.3$ \\
\hline Normal group & 10 & $39.3 \pm 2.3 *$ & $26.1 \pm 2.6^{*}$ & $1.6 \pm 0.3$ & & & \\
\hline
\end{tabular}

$* \mathrm{P}<0.05$.

Table 3. Comparison of IL-10 and M-CSF.

\begin{tabular}{|c|c|c|c|c|c|}
\hline \multirow[t]{2}{*}{ Group } & \multirow[t]{2}{*}{ No. of patients } & \multicolumn{2}{|c|}{ Before treatment } & \multicolumn{2}{|c|}{ After treatment } \\
\hline & & IL-10 & M-CSF & IL-10 & M-CSF \\
\hline Experimental group & 28 & $0.18 \pm 0.04$ & $102.60 \pm 8.40$ & $0.25 \pm 0.01^{\Delta}$ & $116.20 \pm 7.60^{\Delta}$ \\
\hline Control group & 25 & $0.18 \pm 0.03^{\boldsymbol{\nabla}}$ & $102.40 \pm 7.20^{\mathbf{v}}$ & $0.25 \pm 0.02$ & $115.90 \pm 8.40^{\Delta}$ \\
\hline Normal group & 10 & $0.25 \pm 0.03^{+}$ & $116.83 \pm 9.30^{+}$ & & \\
\hline
\end{tabular}

\section{DISCUSSION}

Studies of reproductive immunology indicate that a lack of Ab1 and/or Ab2 leads to insufficient immune protection of the embryo and immune system dysfunction in the mother; this leads to an immune attack from the mother on the fetus and consequently abortion (Sun et al., 1998). Lower levels of IL-10 and M-CSF were recently found to be some of the reasons for this; this may be because decreasing IL-10 levels restrict Th1 response, whereas excess levels lead to abortion. Meanwhile, low levels of M-CSF retard trophoblast proliferation and lead to abortion (Yan et al., 2006). In the present study, the IL-10 and M-CSF levels of the experimental and control groups were lower than those of the normal group $(\mathrm{P}<0.05)$. This indicates patients with RSA lacking blocking antibodies have lower levels of IL-10 and MCSF, which is concordant with the literature. In turn, this further indicates decreases in IL-10 and M-CSF are causes of RSA.

Traditional Chinese medicine has long recognized the need for fetal protection. As the main ingredients of this decoction, human placenta, Morinda officinalis, and Epimedium herb tonify the kidneys, strengthen yang, nourish the blood, and boost essence. Meanwhile, 
Cuscuta chinensis, Dipsacaceae, Eucommia cortex (Eucommiae) also nourish the blood, boost essence, strengthen yang as well as strengthen the bones and muscles. Astragalus, large-headed Atractylodis, Codonopsis pilosula and white peony root strengthen the spleen, solidify yin, and nourish the blood as adjuvants. Furthermore, Glycyrrhiza uralensis benefits qi, strengthens the spleen, and blends the other ingredients. These medicines collectively warm the kidneys, strengthen the spleen, nourish the blood, and protect the fetus. This decoction regulates the immune and endocrine systems in general as well as each aspect listed above. The results of this study indicate after treatment with tiaomian III decoction, the positive conversion rate of $\mathrm{Ab} 1$ and $\mathrm{Ab} 2$ reached 87.1\%; IL-10, M-CSF, and CD8 levels increased significantly; and the 1 -year pregnancy rate was $58.1 \%$. Thus, this decoction promotes the formation of blocking antibodies and cytokine secretion, effectively maintaining pregnancy. Furthermore, it has a relatively strong clinical on RSA lacking blocking antibodies.

\section{REFERENCES}

Gao HY, Tao EX, and Wang Y. (2012) Immunoregulation effects of Tiaomian No. 3 for recurrent spontaneous abortion caused by shortage of blocking antibodies. Zhongguo Zhong Xi Yi Jie He Za Zhi. 32: 766-9.

Sun XX, Li DJ, Zhu Y, Wang YY, et al. (1998). Closed antibodies type anti unique application value in the repeated spontaneous abortion. Zhong Guo Mian Yi Xue Za Zhi. 14: 424.

Yan H, Liu C, Yang SJ, Bao XS, et al. (2006). Unknown cause repeated spontaneous abortion patients before and after initiative immunotherapy of serum interleukin-10, macrophage colony stimulating factor, leukemia inhibitory factor concentration change. Sheng Zhi Yi Xue Za Zhi. 15: 22-25.

Yu J, Li DJ, Zhu Y, Xie X, et al. (2002). Repeated spontaneous abortion immune etiology classification and meaning. Zhong Guo Mian Yi Xue Za Zhi. 18: 117-117. 